\title{
Perception Survey of Sedation Therapy
}

\author{
Lee Sun Kyoung ${ }^{1}$, Seong Jeong Min ${ }^{2, *}$ \\ ${ }^{1}$ Department of Dental Technology, Kyungdong University \\ ${ }^{2}$ Department of Dental Hygiene, Kangwon National University
}

\begin{abstract}
Objectives: In children with incomplete emotional development, anxiety and fear of dental treatment cause very extreme and uncooperative behavior that makes dental treatment impossible (Lee Sang Min et al, THE EFFECTIVENESS OF TWO SEDATIVE REGIMEN USED FOR CHILD PATIENT WHO FAILED TO ORAL CHLORAL HYDRATE, Journal of the Korean academy of pediatric dentistry v.27 no.4, 2000, pp.505-516). It is often used in anesthesia, which is commonly used in pediatric dentistry. The degree of sedation typically required in dental clinical practice is three levels of subconsciousness, the level of hypertrophic, shallow sleep suggested by the American Academy of Pediatrics (AAPD) (http://www. kapd.org). In the past, it could be seen in pediatric dentistry, but in recent years, sedation therapy is becoming more common at dental hospitals and clinics for both children and adults. Therefore, in this study, the awareness and satisfaction of sedation methods were identified for patients who visited a dental hospital in Seoul, and by finding ways to improve their awareness, the purpose of this study was to become a guideline that could be used for dental treatment and dental treatment assistance.
\end{abstract}

Methods: A self-filling survey method was conducted, and 96 of the 120 distributed questionnaires were recovered, and 90 copies were selected as final research subjects, excluding 6 copies with insincere responses to the questionnaire. As a research tool, the structured questionnaire was modified and supplemented to fit this study (Choi Young Shim et al, SEDATION PRACTICES IN DENTAL OFFICE : A SURVEY OF MEMBERS OF THE KOREAN ACADEMY OF PEDIATRIC DENTISTRY, Journal of the Korean academy of pediatric dentistry v.26 no.4 , 1999, pp.579-588). The questionnaire consisted of demographic characteristics, satisfaction with sedation therapy, and perception of sedation therapy. Cronbach's was 0.81 . For statistical analysis, frequency analysis and t-test were performed using SPSS (Statistical Package for Social Science) Ver.22.0 statistical program.

Results: Gender-specific sedation satisfaction levels showed that men were more satisfied than women in most categories. Recognition of types of sedation therapy was highest $73.1 \%$. And $51.0 \%$ said they felt anxious about sedation treatment during dental treatment, $37.0 \%$ said they did not feel it, and $12.0 \%$ said most respondents felt anxious during dental treatment.

Conclusions: In the past, dental sedation therapy was a method commonly used in pediatric dentistry for patients or children who refused dental treatment, and was a helpful method to treat while maintaining consciousness. This sedative therapy reduces the patient's anxiety, which can reduce the stress of dental fear treatment and can be said to be an advantage. Therefore, if this study is further expanded and conducted, it will be helpful when systematic education and promotion of sedation therapy is carried out.

Keywords: Dental, Dental Treatment, Sedation Therapy

Copyright (C) 2021. Korean Academy of Preventive Dentistry. All rights reserved.

This is an Open Access article distributed under the terms of the Creative Commons Attribution Non-Commercial License (http://creativecommons.org/licenses/ by-nc/4.0) which permits unrestricted non-commercial use, distribution, and reproduction in any medium, provided the original work is properly cited. 